\title{
Fungos anamórficos (Hyphomycetes) no Semi-árido do Estado da Bahia, Brasil
}

\author{
Flavia Rodrigues Barbosa ${ }^{1,2}$, Luís Fernando Pascholati Gusmão ${ }^{1,3}$ e Fabio Fernandes Barbosa ${ }^{1,2}$
}

\author{
Recebido em 3/04/2006. Aceito em 16/04/2007
}

\begin{abstract}
RESUMO - (Fungos anamórficos (Hyphomycetes) no Semi-árido do Estado da Bahia, Brasil). Durante o levantamento de fungos anamórficos associados à folhas em decomposição de diferentes plantas na região semi-árida do Estado da Bahia, 23 espécies, pertencentes a 19 gêneros foram encontradas. Dessas, uma espécie constitui novo registro para a Bahia e três para o Brasil, respectivamente: Drechslera victoriae (F. Meehan \& H.C. Murphy) Subram. \& B.L. Jain, Ochroconis crassihumicola (Matsush.) de Hoog \& Arx, Pyricularia caffera Matsush. e Tretopileus sphaerophorus (Berk \& M.A. Curtis) Hughes \& Deighton. Descrições, comentários e ilustrações são apresentados para essas espécies.
\end{abstract}

Palavras-chave: biodiversidade, taxonomia, microfungos, folhedo, decomposição

ABSTRACT - (Anamorphic fungi (Hyphomycetes) in the Semi-arid Region of Bahia State, Brazil). During a survey of the anamorphic fungi associated with leaf litter of different plants from the semi-arid region of Bahia state, 23 species belonging to 19 genera were found. One species is recorded for the first time for Bahia and three for Brazil, respectively: Drechslera victoriae (F. Meehan \& H.C. Murphy) Subram. \& B.L. Jain, Ochroconis crassihumicola (Matsush.) de Hoog \& Arx, Pyricularia caffera Matsush. and Tretopileus sphaerophorus (Berk \& M.A. Curtis) Hughes \& Deighton. Descriptions, comments and illustrations are provided for these species.

Key words: biodiversity, taxonomy, microfungi, leaf litter, decomposition

\section{Introdução}

Presentes em diferentes ambientes, os fungos, através da ação de várias enzimas, atuam como decompositores da matéria orgânica (Grandi \& Gusmão 1998). Como decompositores, facilitam a reincorporação de nutrientes ao solo, o que constitui papel importante para a manutenção do equilíbrio dos ecossistemas através da ciclagem de nutrientes (Heredia-Abarca 1994).

Os fungos anamórficos são encontrados na natureza na sua forma assexual, caracterizados por estruturas tais como conidióforos, células conidiogênicas e conídios. Estes últimos têm como função a dispersão e a perpetuação das espécies sendo de grande importância ecológica e taxonômica. Contudo, em alguns casos, os fungos anamórficos podem apresentar a fase sexual (teleomorfa), na maioria conectada a representantes do Filo Ascomycota e alguns do Filo Basidiomycota (Kirk et al. 2001).

A região semi-árida brasileira compreende uma área de 857.999,3 km² (Sudene 1997), abrangendo o norte de Minas Gerais e quase todos os estados do Nordeste, excluindo-se o Maranhão. Nessa região o aspecto fitofisionômico predominante é a caatinga, ocorrendo outros tipos vegetacionais como matas úmidas, matas estacionais, cerrados, tabuleiros e campos rupestres. Estudos sobre fungos anamórficos decompositores do folhedo nos ecossistemas brasileiros são escassos, como salientado por Grandi (1991). Maia \& Gibertoni (2002), em inventário da diversidade de fungos no semi-árido nordestino apresentaram um check list com 451 espécies distribuídas entre os Filos Ascomycota, Basidiomycota, Oomycota, Zygomycota (incluindo a ordem Glomales, atualmente Glomeromycota) e os fungos anamórficos, sendo estes representados por 198 espécies distribuídas em 82 gêneros. Gusmão, comunicação pessoal, em levantamento bibliográfico e de herbários na mesma região observou a ocorrência de 437 espécies de fungos anamórficos distribuídos em 188 gêneros. Esse número ainda pode ser considerado pequeno quando comparado ao número de espécies descritas, cerca de 80.000 (Kirk et al. 2001) e à estimativa de 1,5 milhão

\footnotetext{
1 Universidade Estadual de Feira de Santana, Departamento de Ciências Biológicas, Laboratório de Micologia (LAMIC), BR 116, Km 03, C. Postal 252, 44031-460 Feira de Santana, BA, Brasil

2 Bolsistas/DTI/IMSEAR/CNPq

3 Autor para correspondência: lgusmao@uefs.br
} 
de espécies fúngicas em todo o mundo (Hawksworth 2001).

O presente trabalho teve como objetivo realizar um inventário de Hyphomycetes associados a folhas em decomposição de plantas que ocorrem no Semiárido do Estado da Bahia, contribuindo para o aumento do conhecimento sobre a micodiversidade no Brasil.

\section{Material e métodos}

Dentro do Projeto IMSEAR (Instituto Milênio do Semi-árido), no período de abril a setembro/2002, foram realizadas coletas nos municípios de Caetité, Carinhanha, Gentio do Ouro, Maracás, Morro do Chapéu, Mundo Novo, Santo Inácio, Sento Sé e Xiquexique no Estado da Bahia. Folhas em decomposição de diferentes espécies vegetais foram coletadas e passaram pela técnica de lavagem sucessiva de substratos, descrita detalhadamente por Grandi \& Gusmão (1998). Após a lavagem, os fragmentos foliares foram cortados em pedaços menores, colocados em câmaras-úmidas deixadas em temperatura ambiente $\left(25^{\circ} \mathrm{C}\right)$ durante 45 dias. Nesse período, as estruturas foram retiradas com o auxílio de agulha fina em estereomicroscópio e colocadas diretamente entre lâmina e lamínula com meios de montagem PVL (álcool polivinílico + ácido lático + fenol) e PVL + azul de algodão. A identificação foi feita em microscópio óptico, por meio de mensurações das estruturas como: conídio, célula conidiogênica, conidióforo, setas, sétulas, etc. Após identificação, as lâminas foram depositadas no Herbário da Universidade Estadual de Feira de Santana (HUEFS). As espécies registradas pela primeira vez para o Estado da Bahia e para o Brasil foram descritas e ilustradas em detalhes. Para as demais foram apresentadas referências de descrição e ilustração e a distribuição geográfica.

\section{Resultados e discussão}

Foram identificados 23 táxons correspondentes a 19 gêneros de Hyphomycetes associados à decomposição de folhas coletadas na região semi-árida do Estado da Bahia. Drechslera victoriae (F. Meehan \& H.C. Murphy) Subram. \& B.L. Jain é referida ela primeira vez para a Bahia, enquanto Ochroconis crassihumicola (Matsush.) de Hoog \& Arx, Pyricularia caffera Matsush. e Tretopileus sphaerophorus (Berk \& Curt.) Hughes \& Deighton são referidas pela primeira vez para o Brasil.
Ardhachandra cristaspora (Matsush.) Subram. \& Sudha, Can. J. Bot. 56(7): 73. 1978.

Basiônimo: Rhinocladiella cristaspora Matsush., Microfungi of the Solomon Islands and Papua-New Guinea: 49. 1971.

Descrição e ilustração: Grandi \& Gusmão (2002, como R. cristaspora).

Material examinado: BRASIL. Bahia: Gentio do Ouro, 16/VII/2002, sobre folhas em decomposição de Hymenaea martiana (Caesalpiniaceae), Gusmão s.n. (HUEFS-56735).

Distribuição geográfica: Austrália (Matsushima 1989), Brasil (Bahia e São Paulo), Costa do Marfim, Tanzânia, Zâmbia (Onofri \& Castagnola 1983), Cuba, Espanha, Índia, Japão, Papua-Nova Guiné, Peru, Taiwan (Grandi \& Gusmão 2002).

Beltrania rhombica Penz., Nuovo G. Bot. Ital. 14: 72. 1882.

Descrição e ilustração: Gusmão \& Grandi (1996). Material examinado: BRASIL. Bahia: Gentio do Ouro, 16/VII/2002, sobre folhas em decomposição de Hymenaea martiana (Caesalpiniaceae), Gusmão s.n. (HUEFS-56764).

Distribuição geográfica: cosmopolita (Gusmão \& Grandi 1996).

Cladosporium cladosporioides (Fresen.) G.A. de Vries, Contribution to Knowledge of the Genus Cladosporium Link ex Fries: 57. 1952.

Basiônimo: Penicillium cladosporioides Fresen., Beitr. Mykol. 3: 22. 1850.

Descrição e ilustração: Grandi \& Attili (1996).

Material examinado: BRASIL. Bahia: Caetité, 16/VII/2002, sobre folhas em decomposição de Asteraceae, Gusmão s.n. (HUEFS-56791); Maracás, 4/IX/2002, sobre folhas em decomposição de Croton sp. (Euphorbiaceae), Gusmão s.n. (HUEFS-56802).

Distribuição geográfica: cosmopolita (Ellis 1971).

Cladosporium oxysporum Berk. \& M. A. Curtis, J. Linn. Soc. Bot. 10: 362. 1868.

Descrição e ilustração: Grandi \& Gusmão (1996). Material examinado: BRASIL. Bahia: Caetité, 21/V/2002, sobre folhas em decomposição de Stachytarpheta aff. quadrangula (Verbenaceae), Gusmão s.n. (HUEFS-56788), 20/V/2002, sobre folhas em decomposição de Hyptis crinita (Lamiaceae), Gusmão s.n. (HUEFS-56789), 16/V/2002, sobre folhas em decomposição de Asteraceae, Gusmão s.n. 
(HUEFS-56790); Gentio do Ouro, 16/VII/2002, sobre folhas em decomposição de Hymenaea martiana (Caesalpiniaceae), Gusmão s.n. (HUEFS-56787), 27/V/2002, sobre folhas em decomposição de Phryngilanthus sp. (Loranthaceae), Gusmão s.n. (HUEFS-56786), 10/VI/2002, sobre folhas em decomposição de Cordia trichotoma (Boraginaceae), Gusmão s.n. (HUEFS-56793), 10/VI/2002, sobre folhas em decomposição de Lafoensia sp. (Lythraceae), Gusmão s.n. (HUEFS-56794); Maracás, 19/VIII/2002, sobre folhas em decomposição de Croton sp. (Euphorbiaceae), Gusmão s.n. (HUEFS56801); Morro do Chapéu, 20/V/2002, sobre folhas em decomposição de Turbina corymbosa (Convolvulaceae), Gusmão s.n. (HUEFS-56799), 30/IV/2002, sobre folhas em decomposição de Annonaceae, Gusmão s.n. (HUEFS-56796); Mundo Novo, 8/IX/2002, sobre folhas em decomposição de Trigonia nivea (Trigoniaceae), Gusmão s.n. (HUEFS-56800); Santo Inácio, 29/VIII/2002, sobre folhas em decomposição de Banisteriopsis sp. (Malpighiaceae), Gusmão s.n. (HUEFS-56792), 16/VII/2002, sobre folhas em decomposição de Pteron sp. (Fabaceae), Gusmão s.n. (HUEFS-56797), 5/VIII/2002, sobre folhas em decomposição de Pavonia glazovianum (Malvaceae), Gusmão s.n. (HUEFS-56798), 2/V/2002, sobre folhas em decomposição de Senna alata (Caesalpiniaceae), Gusmão s.n. (HUEFS56804); Sento Sé, 23/IV/2002, sobre folhas em decomposição de Ipomoea incarnata (Convolvulaceae), Gusmão s.n. (HUEFS-56784), 23/IV/2002, sobre folhas em decomposição de Mansoa sp. (Bignoniaceae), Gusmão s.n. (HUEFS-56785), 30/IV/2002, sobre folhas em decomposição de Tocoyena formosa (Rubiaceae), Gusmão s.n. (HUEFS-56795); Xique-xique, 23/VII/2002, sobre folhas em decomposição de Aspidosperma pyrifolium (Apocynaceae), Gusmão s.n. (HUEFS-56803), 22/VII/2002, sobre folhas em decomposição de Ruellia incompta (Acanthaceae), Gusmão s.n. (HUEFS56805).

Distribuição geográfica: África do Sul, Brasil (Bahia e São Paulo), Canadá, Cuba, El Salvador, Equador, EUA, Índia, Japão (Gusmão, dados não publicados; Grandi 1998).

Drechslera victoriae (F. Meehan \& H.C. Murphy) Subram. \& B.L. Jain, Curr. Sci. 35: 355. 1966.

Fig. 1-3

Basiônimo: Helminthosporium victoriae F. Meehan \& H.C. Murphy, Science 104: 413. 1946.
Teleomorfo: Cochliobolus victoriae R. R. Nelson, Phytopathology 50: 775. 1960.

Conidióforo macronematoso, mononematoso, flexuoso, septado, simples, liso, levemente nodoso, castanho-claro, 288-350×5,7-8,9 $\mu \mathrm{m}$; célula conidiogênica politrética distribuída ao longo do conidióforo, integrada, com proliferação percurrente, cicatrizada, lisa; conídio solitário, 6-10 pseudoseptado, obclavado, reto ou curvo, simples, liso, hilo pouco protuberante, castanho-claro, 45,7-85,4×13,3-19 $\mu \mathrm{m}$.

Material examinado: BRASIL. Bahia: Xiquexique, 18/VII/2002, sobre folhas em decomposição de Aspidosperma pyrifolium (Apocynaceae), Gusmão s.n. (HUEFS-56740).

Distribuição geográfica: cosmopolita (Farr 2007).

O material examinado apresentou características e mensurações que concordaram com Ellis (1971). Essa espécie diferencia-se de Drechslera sorokiniana (Sacc.) Subram. \& B.L. Jain por apresentar conídios mais claros e mais estreitos (Ellis 1971). No Brasil a espécie foi registrada como Bipolaris victoriae ocorrendo em sementes de Avena sativa L. (Mendes et al.1998).

Idriella lunata P.E. Nelson \& S. Wilh., Mycologia 48(4): 550. 1956.

Descrição e ilustração: Ellis (1971).

Material examinado: BRASIL. Bahia: Morro do Chapéu, 30/V/2002, sobre folhas em decomposição de Turbina corimbosa (Convolvulaceae), Gusmão s.n. (HUEFS-56751); Santo Inácio, 10/IX/2002, sobre folhas em decomposição de Pavonia glazovianum (Malvaceae), Gusmão s.n. (HUEFS-56749); Xiquexique, 17/VII/2002, sobre folhas em decomposição de Ruellia incompta (Acanthaceae), Gusmão s.n. (HUEFS-56750).

Distribuição geográfica: provavelmente cosmopolita (Farr 2007).

Memnoniella echinata (Riv.) Galloway, Trans. Br. Mycol. Soc. 18: 165. 1933.

Basiônimo: Penicillium echinatum Rivolta, Dei Parassiti Vegetali: 451. 1873.

Descrições e ilustrações: Ellis (1971), Jong \& Davis (1976), Mercado-Sierra et al. (1997).

Material examinado: BRASIL. Bahia: Santo Inácio, 2/IX/2002, sobre folhas em decomposição de Senna alata (Caesalpiniaceae), Gusmão s.n. (HUEFS-56743); Xique-xique, 7/VIII/2002, sobre folhas em decomposição de Ruellia incompta 
(Acanthaceae), Gusmão s.n. (HUEFS-56744).

Distribuição geográfica: cosmopolita (Ellis 1971).

Myrothecium leucotrichum (Peck) M.C. Tulloch, Mycol. Pap. 130: 121972.

Basiônimo: Excipula leucotricha Peck, Rep. N.Y. St. Mus. nat. Hist 29: 49. 1878.

Descrições e ilustrações: Tulloch (1972), Matsushima (1989).

Material examinado: BRASIL. Bahia: Caetité, 16/V/2002, sobre folhas em decomposição de Asteraceae, Gusmão s.n. (HUEFS-56763); Santo Inácio, 22/VII/2002, sobre folhas em decomposição de Senna alata (Caesalpiniaceae), Gusmão s.n. (HUEFS-56761); Xique-xique, 17/VII/2002, sobre folhas em decomposição de Ruellia incompta (Acanthaceae), Gusmão s.n. (HUEFS-56762).

Distribuição geográfica: Brasil (Bahia) (Gusmão, dados não publicados), Dinamarca, EUA, Malásia, Nigéria, Nova Zelândia, Serra-Leoa, Uganda (Tulloch 1972).

Neojohnstonia minima Gusmão \& Grandi, Mycotaxon 80: 98. 2001.

Descrição e ilustração: Gusmão \& Grandi (2001).

Material examinado: BRASIL. Bahia: Mundo Novo, 29/VIII/2002, sobre folhas em decomposição de Zanthoxylum cf. nigrum (Rutaceae), Gusmão s.n. (HUEFS-56752).

Distribuição geográfica: Brasil (Bahia) (Gusmão \& Grandi 2001).

Ochroconis crassihumicola (Matsush.) de Hoog \& Arx, Kavaka 1: 57. 1973.

Fig. 4-8

Basiônimo: Scolecobasidium crassihumicola Matsush., Microfungi of the Solomon Islands and Papua-New Guinea: 50. 1971.

Conidióforo macronematoso, mononematoso, flexuoso, septado, ramificado, liso, castanho-claro; célula conidiogênica integrada, com proliferação simpodial, denticulada, com dentículos cilíndricos; conídio solitário, com um septo mediano, constrito no septo, elíptico, simples, seco, levemente verrucoso, castanho-claro, 7,0-12×4,2-6,0 $\mu \mathrm{m}$.

Material examinado: BRASIL. Bahia: Mundo Novo, 13/VIII/2002, sobre folhas em decomposição de Zanthoxylum cf. nigrum (Rutaceae), Gusmão s.n. (HUEFS-56756).

Distribuição geográfica: Cuba (Matsushima 1987, como S. crassihumicola), Papua-Nova Guiné
(Matsushima 1971, como S. crassihumicola).

A descrição do material concorda com o descrito na literatura (Matsushima 1987) apesar de Matsushima 1971 não considerar o conídio constricto no septo. O. crassihumicola foi proposto por de Hoog \& Von Arx (1973) como nova combinação de Scolecobasidium crassihumicola Matsush. Segundo estes autores, as características distintivas entre os dois gêneros são: presença de célula conidiogênica ampuliforme e conídios em forma de $\mathrm{Y}$ ou $\mathrm{T}$ em Scolecobasidium e células conidiogênicas surgindo de um micélio com pequenas ramificações cilíndricas e conídios elipsóides a cilíndricos em Ochroconis. Este é o primeiro registro da espécie para o Brasil.

Ochroconis humicola (Barron \& Busch) de Hoog \& Arx, Kavaka 1: 57. 1973.

Basiônimo: Scolecobasidium humicola G.L. Barron \& L.V. Busch, Can. J. Bot. 40: 83. 1962.

Descrição e ilustração: Gusmão, dados não publicados.

Material examinado: BRASIL. Bahia: Mundo Novo, 5/IX/2002, sobre folhas em decomposição de Trigonia nivea (Trigoniaceae), Gusmão s.n. (HUEFS-56758).

Distribuição geográfica: Alemanha, Austrália, Quênia, Tailândia (Farr 2007), Brasil (Bahia e São Paulo) (Gusmão, dados não publicados; Grandi 1989, como S. humicola), Canadá, EUA, Ilhas Marshall, Índia, Paquistão, Paraguai (Grandi 1999, como S. humicola), Cuba, México (Heredia-Abarca \& Reys-Estebanez 1999), Japão (Matsushima 1975, como S. humicola), Papua-Nova Guiné (Matsushima 1971, como S. humicola), Peru (Matsushima 1993), Taiwan (Matsushima 1980, como S. humicola). Provavelmente cosmopolita.

\section{Paraceratocladium polysetosum Castañeda,} Fungi Cubense II: 9. 1987.

Descrição e ilustração: Gusmão \& Barbosa (2003).

Material examinado: BRASIL. Bahia: Sento Sé, 5/V/2002, sobre folhas em decomposição de Tocoyena formosa (Rubiaceae), Gusmão s.n. (HUEFS-56745).

Distribuição geográfica: Brasil (Bahia) (Gusmão \& Barbosa 2003), Cuba (Castañeda-Ruiz 1987).

Periconia byssoides Pers. ex Mérat, Nouv. Fl. Environs Paris, Ed 2, 1: 18. 1821.

Descrição e ilustração: Ellis (1971).

Material examinado: BRASIL. Bahia: Sento Sé, 
6/V/2002, sobre folhas em decomposição de Tocoyena formosa (Rubiaceae), Gusmão s.n. (HUEFS-56781).

Distribuição geográfica: cosmopolita (Ellis 1971).

Periconia cookei Manson \& M. B. Ellis, Mycol. Pap. 56: 72.1953.

Descrições e ilustrações: Ellis (1971).

Material examinado: BRASIL. Bahia: Caetité, 20/V/2002, sobre folhas em decomposição de Hyptis crinita (Lamiaceae), Gusmão s.n. (HUEFS-56779), 16/V/2002, sobre folhas em decomposição de Asteraceae, Gusmão s.n. (HUEFS-56780); Gentio do Ouro, 27/VI/2002, sobre folhas em decomposição de Hymenaea martiana (Caesalpiniaceae), Gusmão s.n. (HUEFS-56782); Morro do Chapéu, 2/V/2002, sobre folhas em decomposição de Turbina corymbosa (Convolvulaceae), Gusmão s.n. (HUEFS-56783).

Distribuição geográfica: Brasil (Bahia) (Gusmão, dados não publicados), Flórida (Fell \& Hunter 1979), Hungria (Révay 1998), Inglaterra (Mason \& Ellis 1953), Taiwan (Matsushima 1980). Provavelmente cosmopolita.

Pithomyces chartarum (Berk. \& M. A. Curtis) Ellis, Mycol. Pap. 76: 13. 1960.

Basiônimo: Sporidesmium chartarum Berk. \& M. A. Curtis, Grevillea 3(26): 50. 1874.

Descrição e ilustração: Grandi (1991).

Material examinado: BRASIL. Bahia: Gentio do Ouro, 10/VI/2002, sobre folhas em decomposição de Cordia trichotoma (Boraginaceae), Gusmão s.n. (HUEFS-56769); Sento Sé, 30/IV/2002, sobre folhas em decomposição de Tocoyena formosa (Rubiaceae), Gusmão s.n. (HUEFS-56770), 2/V/2002, sobre folhas em decomposição de Dioclea grandiflora (Fabaceae), Gusmão s.n. (HUEFS-56771).

Distribuição geográfica: cosmopolita (Ellis 1971).

Pyricularia caffera Matsush., Matsushima Mycological Memoirs 9: 23. 1996.

Fig. 9-11

Conidióforo macronematoso, mononematoso, flexuoso, septado, não ramificado, liso, nodoso, castanho-oliváceo, 105-135×2,3-3 $\mu \mathrm{m}$; célula conidiogênica poliblástica, intercalar, integrada, com proliferação simpodial, denticulada, com dentículos cilíndricos; conídio solitário, 1-septado, naviculado, constricto no septo, seco, verrucoso, com hilo protuberante, hialino, $7,5-15 \times 3 \mu \mathrm{m}$.

Material examinado: BRASIL. Bahia: Caetité, 16/V/2002, sobre folhas em decomposição de
Asteraceae, Gusmão s.n. (HUEFS-56748).

Distribuição geográfica: África do Sul (Matsushima 1996).

O material observado apresentou conídios menores quando comparado à descrição de Matsushima (1996), porém as demais características permitiram identificar como $P$. caffera. A espécie foi isolada de folhas mortas submersas de Aceris sp. na África do Sul (Matsushima 1996). Este é o primeiro registro da espécie para o Brasil.

Selenosporella curvispora MacGarvie, Sci. Proc. R. Dubl. Soc., Ser. B 2: 153. 1968.

Descrição e ilustração: Ellis (1971).

Material examinado: BRASIL. Bahia: Carinhanha, 17/V/2002, sobre folhas em decomposição de Caesalpiniaceae, Gusmão s.n. (HUEFS-56755); Morro do Chapéu, 30/IV/2002, sobre folhas em decomposição de Annonaceae, Gusmão s.n. (HUEFS56754).

Distribuição geográfica: Austrália, França, Índia, Inglaterra, Irlanda, Itália (Gusmão et al. 2001), Brasil (Bahia e São Paulo) (Gusmão, dados não publicados; Gusmão et al. 2001), Japão (Matsushima 1975), México (Heredia-Abarca et al. 1995), Peru (Matsushima 1993). Provavelmente cosmopolita.

Speiropsis scopiformis Kuthub. \& Nawawi, Trans. Br. Mycol. Soc. 89: 584. 1987.

Descrição e ilustração: Barbosa \& Gusmão (2005).

Material examinado: BRASIL. Bahia: Gentio do Ouro, 16/VII/2002, sobre folhas em decomposição de Hymenaea martiana (Caesalpiniaceae), Gusmão s.n. (HUEFS-56776); Santo Inácio, 19/VIII/2002, sobre folhas em decomposição de Banisteriopsis sp. (Malpighiaceae), Gusmão s.n. (HUEFS-56777); Mundo Novo, 13/VIII/2002, sobre folhas em decomposição de Zanthoxylum cf. nigrum (Rutaceae), Gusmão s.n. (HUEFS-56775).

Distribuição geográfica: Brasil (Bahia e São Paulo) (Gusmão et al. 2001; Barbosa \& Gusmão 2005), China (Farr 2007), Cuba, Malásia, Nova Caledônia (Gusmão et al. 2001), México (Heredia-Abarca et al. 2000).

Stachybotrys chartarum (Ehrenb.) Hughes, Can. J. Bot. 36: 812. 1958.

Basiônimo: Stilbospora chartarum Ehrenb., Sylv. Mycol. Berol. 9: 21. 1818.

Descrições e ilustrações: Matsushima (1975), Jong \& Davis (1976), Mercado-Sierra et al. (1997).

Material examinado: BRASIL. Bahia: Caetité, 
16/V/2002, sobre folhas em decomposição de Asteraceae, Gusmão s.n. (HUEFS-56746).

Distribuição geográfica: Arábia Saudita, Austrália, Canadá, Cuba, EUA, Hawaii, Índia, Inglaterra, Japão, Taiwan (Whitton et al. 2001), Brasil (Bahia) (Gusmão, dados não publicados).

Stachybotrys nephrospora Hansf., Proc. Linn Soc. London 155: 45.1943.

Descrições e ilustrações: Jong \& Davis (1976), Mercado-Sierra et al. (1997).

Material examinado: BRASIL. Bahia: Gentio do Ouro, 10/VI/2002, sobre folhas em decomposição de Cordia trichotoma (Boraginaceae), Gusmão s.n. (HUEFS-56747).

Distribuição geográfica: Brasil (Bahia) (Gusmão, dados não publicados), Canadá, Cuba, Nigéria, Paquistão (Mercado-Sierra et al. 1997), EUA, Tailândia (Farr 2007), Índia, Jamaica, Serra-Leoa (Ellis 1971), Japão, Uganda (Jong \& Davis 1976), PapuaNova Guiné (Matsushima 1971).
Tretopileus sphaerophorus (Berk. \& M.A. Curtis) Hughes \& Deighton, Mycol. Pap. 78: 2. 1960.

Fig. 12-13

Basiônimo: Monotospora sphaerophora Berk. \& M.

A. Curtis, J. Linn. Soc., Bot. 10: 360. 1868.

Conidioma em sinema, cilíndrico, castanho-claro a escuro, 356-581×25,9-74,7 $\mu \mathrm{m}$; hifa do estipe castanho-clara a escura, 2,0-3,0 $\mu \mathrm{m}$ larg.; bulbilhos multicelulares, mais ou menos esféricos, bordas irregulares, castanho-escuros, 53-98,5 $\mu$ m diâm.

Material examinado: BRASIL. Bahia: Sento Sé, 6/V/2002, sobre folhas em decomposição de Tocoyena formosa (Rubiaceae), Gusmão s.n. (HUEFS-56772).

Distribuição geográfica: Bahamas, Cuba, Indonésia, Paraguai (Seifert 1990), EUA, Java, México, Serra-Leoa (Heredia-Abarca et al. 2000), Malásia, Tailândia, Taiwan (Matsushima 1995).

A morfologia peculiar da espécie permite sua fácil identificação. A função dos bulbilhos ainda permanece desconhecida (Heredia-Abarca et al. 2000). Segundo

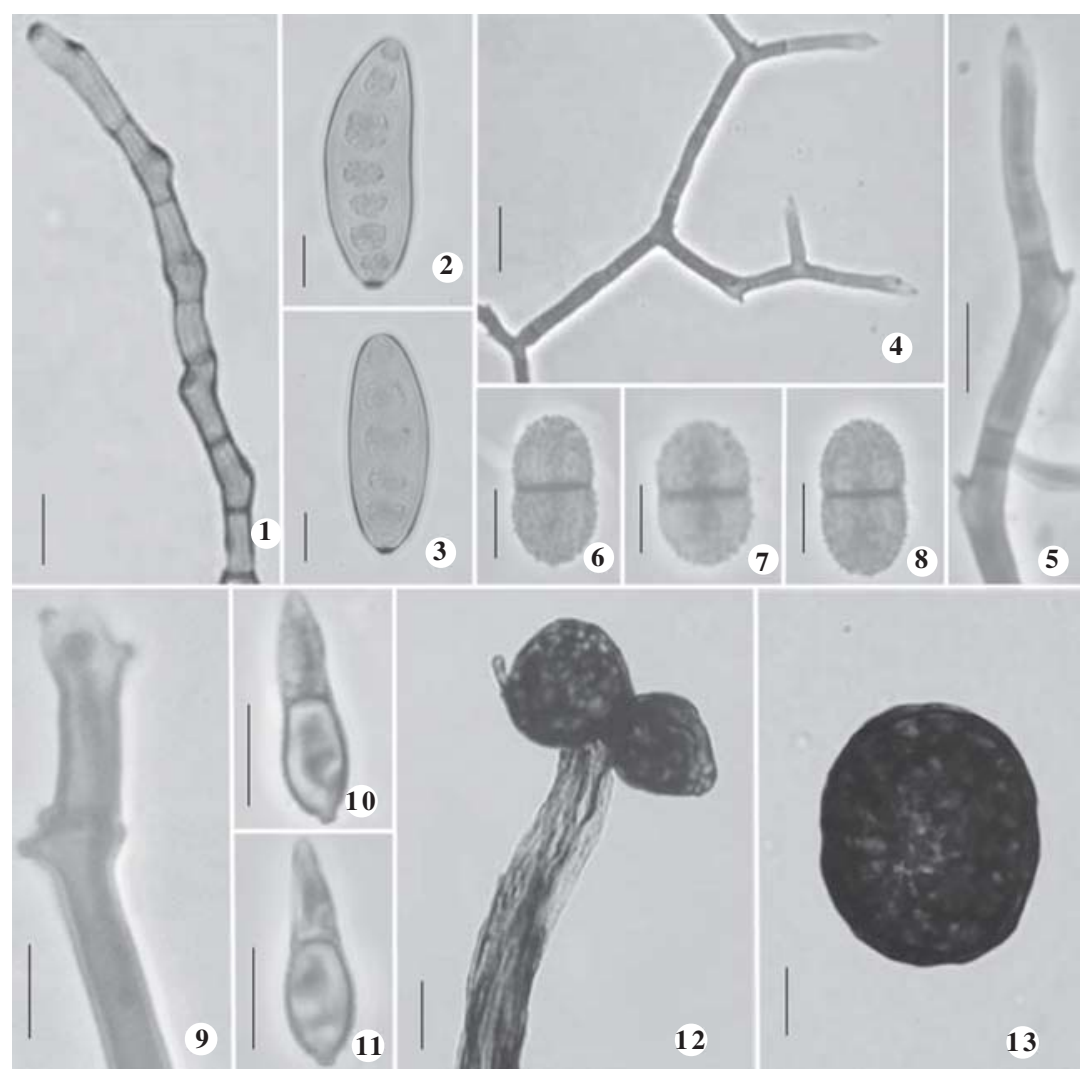

Figuras 1-13: 1-3. Drechslera victoriae (F. Meehan \& H.C. Murphy) Subram. \& B.L. Jain. 1. Detalhe do ápice do conidióforo; 2-3. Conídios. 4-8. Ochroconis crassihumicola (Matsush.) de Hoog \& Arx. 4. Visão geral do conidióforo; 5. Detalhe do ápice do conidióforo; 6-8. Conídios. 9-11. Pyricularia caffera Matsush. 9. Detalhe do conidióforo; 10-11. Conídios. 12-13. Tretopileus sphaerophorus (Berk. \& M.A. Curtis) Hughes \& Deighton. 12. Conidioma sinematoso. 13. Bulbilho. Barras $=1 \quad(40 \mu \mathrm{m}) ; 2-4(10 \mu \mathrm{m}) ; 5-11=(5 \mu \mathrm{m})$; $12(50 \mu \mathrm{m}) ; 13(20 \mu \mathrm{m})$. 
Matsushima (1995) esta espécie é considerada como fase anamorfa de basidiomiceto devido à presença de fíbulas nas hifas do estipe, característica não evidenciada no material examinado. Seifert (1990) foi o primeiro a registrar a ocorrência da espécie no Oriente. Este é o primeiro registro da espécie para o Brasil.

Volutella minima Höhn., Sber. Akad. Wiss. Wien, Math. Naturw. Kl., Abt. 1 118: 1543. 1909.

Descrição e ilustração: Gusmão \& Grandi (1997).

Material examinado: BRASIL. Bahia: Xiquexique, 7/VIII/2002, sobre folhas em decomposição de Ruellia incompta (Acanthaceae), Gusmão s.n. (HUEFS-56734).

Distribuição geográfica: Colômbia, EUA, Java, Nova Zelândia, Panamá, Brasil, Índia, Inglaterra, Peru (Gusmão \& Grandi 1997).

Wiesneriomyces laurinus (Tassi) P.M. Kirk, Trans. Br. Mycol. Soc. 82(4): 748. 1984.

Basiônimo: Volutellaria laurina Tassi [stat. anam.], Atti della Reale Accademia dei Fisiocritici di Siena, Serie 4 8: 551. 1897.

Descrição e ilustração: Gusmão \& Grandi (1997).

Material examinado: BRASIL. Bahia: Gentio do Ouro, 16/VII/2002, sobre folhas em decomposição de Hymenaea martiana (Caesalpiniaceae), Gusmão s.n. (HUEFS-56759).

Distribuição geográfica: cosmopolita (Gusmão \& Grandi 1997).

\section{Agradecimentos}

Os autores agradecem o suporte financeiro propiciado pelo projeto IMSEAR (Instituto do Milênio do Semi-Árido) e ao CNPq (Conselho Nacional de Desenvolvimento Científico e Tecnológico) pelas Bolsas concedidas (Processos 381296/2004-0 e 382416/2004-0).

\section{Referências bibliográficas}

Barbosa, F.F. \& Gusmão, L.F.P. 2005. Two Speiropsis species (Anamorphic Fungi-Hyphomycetes) from Bahia State, Brazil. Acta Botanica Brasilica 19: 515-518.

Castañeda-Ruiz, R.F. 1987. Fungi Cubenses II. Cuba, Instituto de Investigaciones Fundamentales en Agricultura Tropical.

Ellis, M.B. 1971. Dematiaceous Hyphomycetes. Kew, Commonwealth Mycological Institute.
Farr, D.F.; Rossman, A.Y.; Palm, M.E. \& McCray, E.B. 2007. Fungal Databases, Systematic Botany \& Mycology Laboratory, ARS, USDA. http://nt.ars-grin.gov/ fungaldatabases (Acesso em: 18/01/2007).

Fell, J.W. \& Hunter, I.L. 1979. Fungi associated with the decomposition of the black rush, Juncus roemerianus, in south Florida. Mycologia 71: 322-342

Grandi, R.A.P. 1989. Hyphomycetes decompositores 2. Táxons associados às raízes de Miranta bicolor Ker. Revista Brasileira de Biologia 51: 133-141.

Grandi, R.A.P. 1991. Hyphomycetes decompositores 4. Espécies associadas às raízes de Ctenanthe oppenheimiana Sond. Acta Botanica Brasilica 5: 13-23.

Grandi, R.A.P. 1998. Hyphomycetes decompositores do folhedo de Alchordea triplinervia (Spreng.) Mull. Arg. Hoehnea 25: 133-148.

Grandi, R.A.P. 1999. Hyphomycetes decompositores do folhedo de Euterpe edulis Mart. Hoehnea 26: 87-101.

Grandi, R.A.P. \& Attili, D.S. 1996. Hyphomycetes on Alchornea triplinervia (Spreng.) Müell. Arg. leaf litter from the Ecological Reserve Juréia-Itatins, State of São Paulo, Brazil. Mycotaxon 60: 373-386.

Grandi, R.A.P. \& Gusmão, L.F.P. 1996. Hyphomycetes decompositores de raízes de Calathea zebrina (Sims) Lindl. (Marantaceae), provenientes da Reserva Biológica do Alto da Serra de Paranapiacaba, Santo André, SP, Brasil. Revista Brasileira de Botânica 19: 165-172.

Grandi, R.A.P. \& Gusmão, L.F.P. 1998. A técnica da lavagem sucessiva de substratos de plantas como subsídio para estudos da associação fungo/substrato e diversidade de Hyphomycetes nos Ecossistemas. IV Simpósio de Ecossistemas Brasileiros. ACIESP 104: 80-90.

Grandi, R.A.P. \& Gusmão, L.F.P. 2002. Hyphomycetes decompositores do folhedo de Tibouchina pulchra Cogn. Revista Brasileira de Botânica 25: 79-87.

Gusmão, L.F.P. \& Barbosa, F.F. 2003. Paraceratocladium polysetosum, a new record from Brazil. Mycotaxon 85 : 81-84.

Gusmão, L.F.P. \& Grandi, R.A.P. 1996. Espécies do grupo Beltrania (Hyphomycetes) associadas a folhas de Cedrela fissilis Vell (Meliaceae), em Maringá, PR, Brasil. Hoehnea 23: 91-102.

Gusmão, L.F.P. \& Grandi, R.A.P. 1997. Hyphomycetes com conidioma dos tipos esporodóquio e sinema associados a folhas de Cedrela fissilis (Meliaceae), em Maringá, PR, Brasil. Acta Botanica Brasilica 11: 123-134.

Gusmão, L.F.P. \& Grandi, R.A.P. 2001. A new Neojohnstonia species from Brazil. Mycotaxon 80: 97-100.

Gusmão, L.F.P.; Grandi, R.A.P. \& Milanez, A.I. 2001. Hyphomycetes from leaf litter of Miconia cabussu in the Brazilian Atlantic rain forest. Mycotaxon 79: 201-213.

Hawksworth, D.L. 2001. The magnitude of fungal diversity: the 1.5 million species estimate revised. Mycological Research 105: 1422-1432.

Heredia-Abarca, G. 1994. Hifomicetos dematiaceos en bosque mesofilo de montaña. Registros nuevos para México. Acta Botanica Mexicana 27: 15-32. 
Heredia-Abarca, G.; Arias, R.M. \& Reys-Estebanez, M. 2000. Contribución al conocimiento de los hongos Hyphomycetes de México. Acta Botanica Mexicana 51: 39-51.

Heredia-Abarca, G. \& Reys-Estebanez, M. 1999. Hongos conidiales de bosque mesófilo: Algunas especies foliícolas y de la hojarasca desconocidas para México. Revista Mexicana de Micología 15: 79-88.

Heredia-Abarca, G.; Mercado-Sierra, A. \& Mena-Portales, J. 1995 Conidial fungi from leaf litter in a mesophilic cloud forest of Veracruz, México. Mycotaxon 55: 473-490.

Hoog, G.S. \& Von Arx, J.A. 1973. Revision of Scolecobasidium and Pleurophragmium. Kavaka 1: 50-60.

Jong, S.C. \& Davis, E.E. 1976. Contribution to knowledge of Stachybotrys and Memnoniella in culture. Mycotaxon 3: 409-485.

Kirk, P.M.; Cannon, P.F.; David, J.C. \& Stalpers, J.A. 2001. Ainsworth and Bisby's Dictionary of the Fungi. $9^{\text {th }}$ ed. Wallingford, CABI.

Maia, L.C. \& Gibertoni, T.B. 2002. Fungos registrados no semi-árido nordestino. Pp. 163-176. In: E.V.S.B. Sampaio; A.M. Giulietti; J. Virgínio \& C.F.L.G. Rojas (eds.). Vegetação e Flora da Caatinga. Recife, Associação Plantas do Nordeste, APNE/CNIP.

Mason, E.W. \& Ellis, M.B. 1953. British species of Periconia. Mycological Papers 56: 1-127.

Matsushima, T. 1971. Microfungi of the Solomon Islands and Papua-New Guinea. Publicado pelo autor, Kobe.

Matsushima, T. 1975. Icones Microfungorum a Matsushima Lectorum. Publicado pelo autor, Kobe.

Matsushima, T. 1980. Saprophytic microfungi from Taiwan. Part 1. Hyphomycetes. Matsushima Mycological Memories 1. Published by the author, Kobe.
Matsushima, T. 1987. Matsushima Mycological Memoirs n. 5. Publicado pelo autor, Kobe.

Matsushima, T. 1989. Matsushima Mycological Memoirs n. 6. Publicado pelo autor, Kobe.

Matsushima, T. 1993. Matsushima Mycological Memoirs n. 7. Publicado pelo autor, Kobe.

Matsushima, T. 1995. Matsushima Mycological Memoirs n. 8. Publicado pelo autor, Kobe.

Matsushima, T. 1996. Matsushima Mycological Memoirs n. 9. Publicado pelo autor, Kobe.

Mendes, M.A.S.; da Silva, V.L.; Dianese, J.C.; Ferreira, J.A.S.V.; Santos, C.E.N.; Gomes Neto, E.; Urden, A.F. \& Castro, C. 1998. Fungos em Plantas no Brasil. Brasília, Embrapa, SPI.

Mercado-Sierra, A.; Holubová-Jechová, V. \& Mena-Portales, J. 1997. Hifomicetes demaciáceos de CubaEnteroblásticos. Monografie XXIII, Torino, Museo Regionale di Scienze Naturali.

Onofri, S. \& Castagnola, M. 1983. The genera Ardhachandra and Rhinocladiella, their synonymy. Mycotaxon 18: 337-343.

Révay, A. 1998. Review of the Hyphomycetes of Hungary. Studia botanica hungarica 27-28: 5-74

Seifert, K.A. 1990. Synnematous Hyphomycetes. Memoirs of the New York Botanical Garden 59: 109-154.

Sudene. 1997. Caracterização do semi-árido brasileiro. http://www. asabrasil.org.br (Acesso em: 8/06/2004).

Tulloch, M. 1972. The genus Myrothecium Tode ex. Fr. Mycological Papers 130: 1-41.

Whitton, S.R.; McKenzie, E.H.C. \& Hyde, K.D. 2001. Microfungi on the Pandanaceae: Stachybotrys with three new species. New Zealand Journal of Botany 39: 489-499. 